\title{
Physics monitor
}

Contrary to popular belief, nucleons are not held together by exchanges of single pions. Instead, the dominant attraction could come about through the excitation of 'nucleon isobars' with two-pion exchange (as sketched on the left). This is analogous to the dipoledipole interactions of excited states in the van der Waals forces in molecules (right).

\section{The nucleon-nucleon force}

The interaction between pairs of nucleons, the NN force, is one of the main points of contact between nuclear physics and particle physics. A good understanding of this interaction enables nuclear physicists to better understand and describe the behaviour and properties of nuclear matter, while particle physicists gain a deeper insight into the mechanisms which influence individual particles.

The traditional way of looking at the $\mathrm{NN}$ force is as a series of Yukawa-like single meson exchanges. The observed properties of the interaction require five mesons (pi, rho, omega, phi and sigma), each with its own characteristic behaviour. A long-standing embarrassment has been the light (450 MeV) sigma meson, which has been introduced into the theory to provide a central attractive potential. But no such meson exists!

Two-pion exchange can simulate the required features of sigmaexchange, so that the need to identify with a physical particle is not necessary. Nevertheless the idea of the exchange of a single particle is a useful form of 'shorthand' to describe the required central attraction between pairs of nucleons. The exchange of two pions creates a force directly analogous to the van der Waals force in molecules and enables a deep and satisfying analogy to be drawn between the binding of nuclear matter and the forces at work in molecules.

The figure shows on the right the interaction between two neutral molecules in terms of photon exchange. The first photon induces an electric dipole in one molecule and this dipole transmits a photon to the second molecule, creating another dipole. These two dipoles interact and give rise to the van der Waals forces. The overall effect of these induced dipole-induced dipole

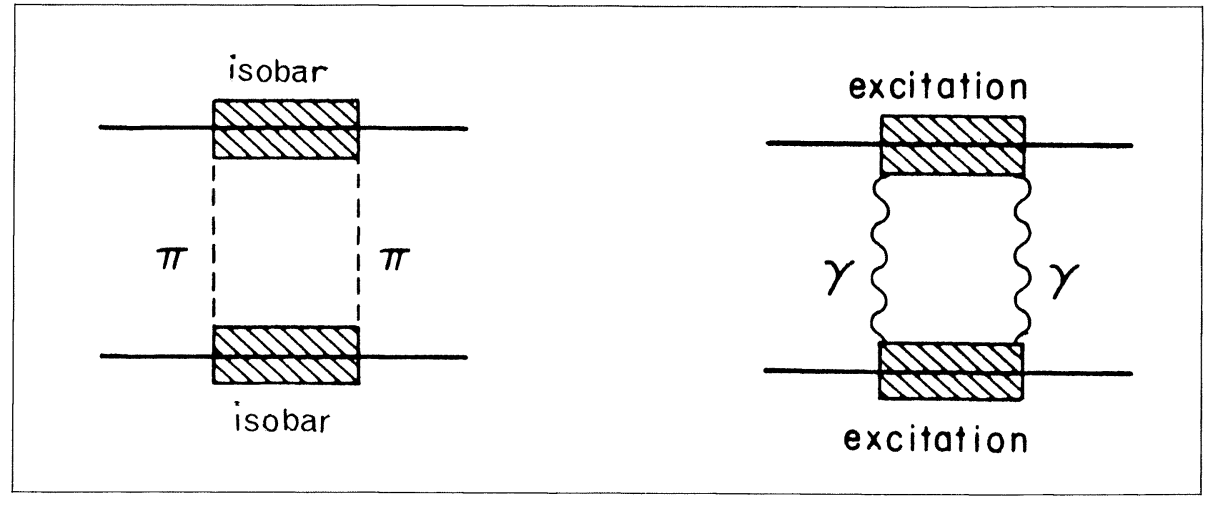

interactions is described by a characteristic structure constant the electric polarizability.

In the nucleon-nucleon case, pions take the place of photons, while the excitation of nucleon isobars corresponds to the creation of induced dipoles. This mechanism gives rise to a medium-range attraction which dominates effects due to single pion exchange. Thus, contrary to popular belief, nuclei are held together by a pionic equivalent of the van der Waals mechanism and not by single pion exchange.

While the nucleon-nucleon interaction is attractive at medium range (a distance greater than about 0.8 fermi), there is a strong repulsion from about 0.5 fermi inwards, interpreted as the result of omega exchange and it is this repulsion which makes the close study of the proton so difficult. It is inside this repulsive core that quarks could exist, confined by a suitable mechanism (see below).

Thus the nucleon-nucleon interaction is well described at relatively long distances while the investigation of the inner regions is more difficult. The single omega exchange, strongly repulsive for the nucleon-nucleon case, becomes attractive between nucleons and antinucleons and could enable bound states to occur (see August issue, page 243). One immediate task is to identify the quantum numbers of such 'baryonium' states and to formulate the correct selection rules.

This study provides more fuel both for quark model investigations and for nuclear dynamics, so extending the valuable common ground between nuclear and particle physicists.

\section{Quark confinement : 1. Quantum chromodynamics}

Despite the impressive list of predictions to its credit, the quark model of hadrons was long overshadowed by the reluctance of the quarks to unashamedly reveal themselves. Now, this reluctance is interpreted as the result of quark 'confinement' - the quarks are there all right, but they find it difficult to leave each other's company.

There are number of ways of trying to explain this permanent confinement of quarks inside hadrons. One, called quantum chromodynamics, is yet another example of the use of gauge theories in particle physics (see September issue, page 271), while a totally different approach sidesteps the problems of field theory and presupposes that the quarks exist only inside a small volume, or 'bag'. Quarks in bags, etc., will be dealt with in a subsequent article. 
In the early days of the quark model, the non-appearance of quarks was explained by supposing that free quarks were extremely heavy, but were bound together by tremendously strong forces which swallowed up most of this rest mass to give relatively light hadrons. This in essence was the first model of quark confinement.

In such a picture of heavy quarks cemented together by the major proportion of their rest mass, one would expect additional quarkantiquark pairs to be created relatively easily, and this is not seen. Also such a picture did not tie in with notions of quark 'additivity', where many good predictions can be made simply by adding together the properties of the constituent quarks in a reaction. Quark model calculations could literally be done on the backs of envelopes and, what's more, gave some good results. If the quarks retained their individuality in strong interaction dynamics to such an extent, it would be difficult to imagine them as being crushed together by some still stronger force.

The next clue came from the classic experiments at SLAC which showed that hadrons probed with high energy electron beams behave like boxes of small, extremely light particles, or 'partons'. Subsequent experiments with neutrino beams at CERN using the Gargamelle bubble chamber showed similar behaviour and demonstrated that these parton constituents inside nucleons had quark-like properties.

These and similar experiments show that when the deep inner structure of the nucleon is probed by weakly and electromagnetically interacting particles, spin $1 / 2$ quarks show up. Analysis shows that the masses of these quarks are negligible compared with the accompanying momentum transfers of $1 \mathrm{GeV}$ or so and under these conditions even the quark-quark interactions seem to become negligible.

This discovery forces us to the conclusion that while free quarks are reluctant to come out into the open, they are nevertheless only loosely bound together in hadrons. Quarks stick together permanently but gently!

\section{Asymptotic freedom}

In a Yang-Mills field theory, the parameters describing internal particle properties (isospin, etc.) are allowed to depend on space and time so that a formalism can be developed which is broadly analogous to the highly successful techniques of quantum electrodynamics.

Several years ago, it was discovered that using such a Yang-Mills field theory within a small enough region of space-time, quarks would not interact very much, a condition known in the trade as 'asymptotic freedom'. If this space-time volume were enlarged, the level of interaction between the quarks would become stronger. This is in complete contrast with quantum electrodynamics, where the effective charge on the electron becomes bigger and bigger as the space-time volume of interaction is decreased, leading eventually to an infinite charge for a 'bare' electron.

This infinity in quantum electrodynamics is naturally removed from the theory by the process of 'renormalisation' which takes account of the additional interactions surrounding a bare electron to give the small observable electronic charge. In Yang-Mills field theory, the corresponding 'bare' charge for the isolated particle is zero, and becomes bigger as the additional surrounding interactions are included.

\section{Colour}

In the same way as electromagnetic interactions are mediated by the exchange of massless spin one photons, so such a field theory of quark-quark interactions says that the interactions are mediated by the exchange of massless spin one particles, called 'gluons'.
An analogous quantity to electric charge must exist in the quark theory, and this is where 'colour' comes in. The gluons carry colour between the quarks, which have a colour charge.

The need for additional quark quantum numbers besides 'flavour' (up, down, strange, charm and whatever else) had been suspected for some time. When the decuplet of heavy baryons (the family which includes the Omega-minus) is constructed from three quarks, it has a wave function which is symmetric both in flavour and angular momentum (spin). However, according to the Pauli exclusion principle, the wave functions of all particles with half-integer spin (including baryons) should be antisymmetric.

This is easily remedied by bringing in another quantum number like 'colour' but the ideas of colour soon take on a deeper significance. In the colour picture, quarks have three additional attributes which like charge, isospin, etc., carry over into hadrons. If there were no restrictions, the colour model would produce all sorts of colour variations of the hadrons built from quarks, but such variations are not seen. (It is important to remember that 'colours' refer only to quantum numbers, and cannot be seen as colours, any more than 'flavours' can be tasted!)

To keep down the number of possible hadrons, we can say that all observed particles have to have zero colour (i.e. they are 'white' in a composite colour picture). For baryons made from three quarks, the zero colour combination is found to be antisymmetric, so if the total baryon wave function is built up from three factors, flavour, angular momentum and colour, then it is indeed antisymmetric as required by the exclusion principle.

Quark interactions must therefore be such that all hadrons have zero colour, and the problem of quark confinement is then put in another way: why cannot colours be separated? The 


\section{People and things}

simplest quark configurations which have zero colour are a quark-antiquark pair (a meson) and a triplet of three quarks (a baryon). Other combinations of quarks having net colour and noninteger quantum numbers are not seen.

\section{Problems}

In principle, the problem of quark confinement and the general behaviour of the quark-quark interaction can be studied using a Yang-Mills field theory which incorporates these ideas of colour. This theory is quantum chromodynamics, frequently abbreviated to QCD.

However, in field theory, the only calculational framework which is well understood is the perturbation technique in which successive levels of interaction are obtained to get a closer and closer approximation to the 'answer'.

In quantum electrodynamics, the effective charge which describes the size of the interaction between a charged particle and the electric field is a small number. A perturbation series expansion therefore has successive terms which get smaller and smaller and this series is easy to handle.

Using these perturbation techniques in quantum chromodynamics, we can handle the small colour charges which occur when quarks are described in small enough regions of space-time, but the effective colour charge quickly increases outside this small regime of asymptotic freedom to give series expansions which cannot be handled.

Using these limited calculational techniques, quantum chromodynamics cannot describe quark confinement. This is not to say that the theory is wrong: it is just that the right mathematical methods for handling the calculations have yet to be found.

Nevertheless, perturbation theory can still be applied in the small spacetime regions around each quark where the effective colour charge is small enough. This is equivalent to treating the hadron as some kind of 'box' of free quarks and, if the idea is applied to neutrino-nucleon interactions, results are obtained which mysteriously agree with experiment!

Thus we arrive at a picture of quark interactions which can only be applied inside small enough regions of spacetime. This does not explain confinement but, nevertheless, gives some results which agree with experiment. This state of affairs is not yet understood, but it does mean that further useful calculations using asymptotic freedom might be possible, given the right kinematical conditions.

Until the mathematical tools available for field theory have been developed further to give a more complete framework for calculations, some people are turning away from this incomplete description of quark interactions in favour of one which presupposes confinement. This 'bag' model will be dealt with in a subsequent article.

\section{On stage}

An experimental theatre group called 'The Phantom Captain' has brought particle physics to the London stage with a work entitled 'The (2nd) Changeness Congress'. Using Nigel

Calder's 'The Key to the Universe' and Fritjof Capra's 'The Tao of Physics' as sources of inspiration, they put together, in cabaret form, a lively (and accurate) presentation of the present turmoil of our understanding of the nature of matter. Robert Walgate, favourably reviewing the event in the 'New Scientist', maintains that The Phantom Captain have made a breakthrough being 'the first group ever to make particle physics sexy'. We are obviously going to pay dearly for the discovery of charm.

\section{CERN School of Physics}

The 1978 CERN School of Physics is being organized in collaboration with the Dutch Physical Society. It will be held from 4-17 June at Austerlitz-Zeist near Utrecht in the Netherlands. The aim of the School is to communicate aspects of current theoretical physics to young experimental physicists mainly from the CERN Member States. Further information may be obtained from Miss D.A. Caton, Scientific Conference Secretariat, CERN, CH1211 Geneva 23, Switzerland.

\section{USA reorganization}

We mentioned in the September issue the creation of the Department of Energy (DOE) in the USA under Secretary of Energy, James Schlesinger. Within the Department there is an Office of Energy Research headed by a Director who reports to the Secretary on the physical research programmes, such as high energy physics, transferred from the former Energy Research and Development Administration (ERDA). The Director of 